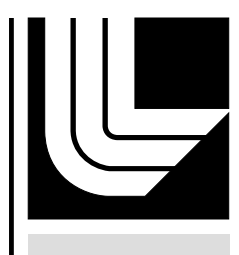

LAW RENCE LIVERMORE N A T IO N A L LABORATORY

\title{
UCRL-TR-210446
}

\section{Direct Probing of Protein-Protein Interactions}

A. Noy, T. A. Sulchek, R. W. Friddle

March 11, 2005 
This document was prepared as an account of work sponsored by an agency of the United States Government. Neither the United States Government nor the University of California nor any of their employees, makes any warranty, express or implied, or assumes any legal liability or responsibility for the accuracy, completeness, or usefulness of any information, apparatus, product, or process disclosed, or represents that its use would not infringe privately owned rights. Reference herein to any specific commercial product, process, or service by trade name, trademark, manufacturer, or otherwise, does not necessarily constitute or imply its endorsement, recommendation, or favoring by the United States Government or the University of California. The views and opinions of authors expressed herein do not necessarily state or reflect those of the United States Government or the University of California, and shall not be used for advertising or product endorsement purposes.

This work was performed under the auspices of the U.S. Department of Energy by University of California, Lawrence Livermore National Laboratory under Contract W-7405-Eng-48. 


\section{Direct probing of protein-protein interactions}

Reconstructions of equilibrium interaction potentials from non-equilibrium force microscopy measurements

Principal Investigator: Aleksandr Noy

Chemistry and Materials Science

Co-Investigators: Todd A. Sulchek,

Biology and Biotechnology Research Program

Raymond W. Friddle

Chemistry and Materials Science

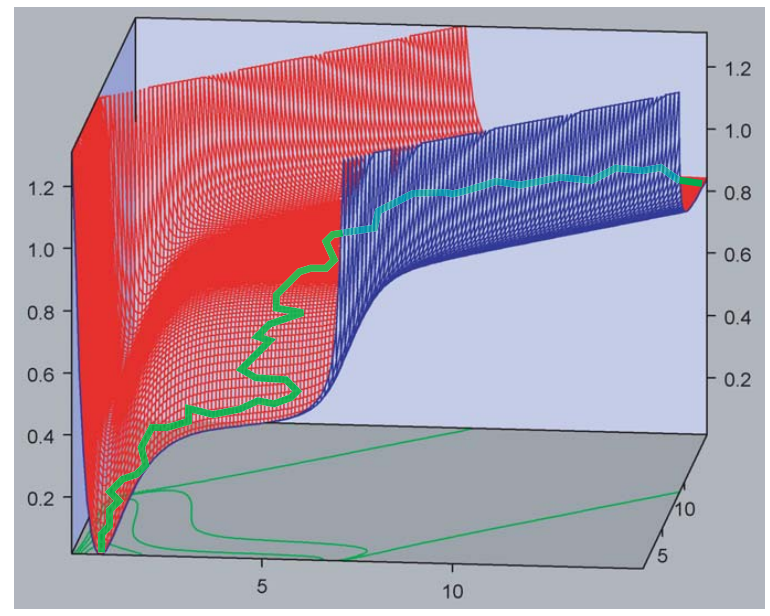

FY04 LDRD FS Final Report

March, 2005 


\section{Contents}

1 Executive Summary 3

2 Background and Theoretical Framework 3

2.1 Direct measurement of intermolecular interactions . . . . . . . . . 3

2.2 Noncovalent chemical bond dynamics under external load . . . . . . 5

2.3 Unbinding far from equilibrium: Tethered ligand-receptor system . . 6

2.4 Near-equilibrium unbinding: Jarzynski equality and reconstruction procedure . . . . . . . . . . . . . . . 8

3 Results 9

3.1 Monte-Carlo simulation of a tethered ligand-receptor system . . . . . 9

3.2 Equilibrium energy profile reconstruction for a short DNA duplex . . 11

3.3 Dynamic Force Spectroscopy of Mucin 1 interactions with single and multiple antibodies . . . . . . . . . . . . . . . 12

3.3.1 Design and preparation of an in-vitro model system for force spectroscopy of multivalent binding of tethered antibodies targeting Muc1 .................... 14

3.3.2 Detection of specific antibody-Muc1 binding . . . . . . . . . . 14

3.3.3 Force spectroscopy determination of kinetic off-rates for mono, bi-, and tri-valent tethered antibodies . . . . . . . . . 15 


\section{Executive Summary}

This project aimed to establish feasibility of using experimental techniques based on direct measurements of interaction forces on the single molecule scale to characterize equilibrium interaction potentials between individual biological molecules. Such capability will impact several research areas, ranging from rapid interaction screening capabilities to providing verifiable inputs for computational models. It should be one of the enabling technologies for modern proteomics research.

This study used a combination of Monte-Carlo simulations, theoretical considerations, and direct experimental measurements to investigate two model systems that represented typical experimental situations: force-induced melting of DNA rigidly attached to the tip, and force-induced unbinding of a protein-antibody pair connected to flexible tethers. Our results establish that for both systems researchers can use force spectroscopy measurements to extract reliable information about equilibrium interaction potentials. However, the approaches necessary to extract these potentials in each case- Jarzynski reconstruction and Dynamic Force Spectroscopyare very different. We also show how the thermodynamics and kinetics of unbinding process dictates the choice between in each case.

The project resulted in a peer-reviewed publication in Applied Physics Letters (A. Noy, Appl. Phys. Lett., v.85(20), p.4792 ). In addition the PI gave an invited tutorial on force spectroscopy at the University of Minnesota based on some of the results of this study. The research team is currently preparing the results on antibody-antigen binding measurements for publication.

\section{Background and Theoretical Framework}

\subsection{Direct measurement of intermolecular interactions}

Biological processes are driven by the interactions between molecular components of cellular machinery, which often give rise to complicated potential energy landscapes. Biological interactions are also unique among other intermolecular interactions, because they often occur under conditions that are far from equilibrium. A vast number of cellular processes are driven by various ionic, chemical and electrostatic potential gradients and often involve substantial unidirectional fluxes of ions and molecules. Applying and measuring the mechanical forces that extend and rupture molecular bonds gives us a unique control parameter that allows us to explore potential energy surfaces, and even simulate dissipative conditions by controlling the loading kinetics.

Last decade saw the development of molecular force measurement techniques, mainly atomic force microscopy and optical trapping, which gave researchers robust 


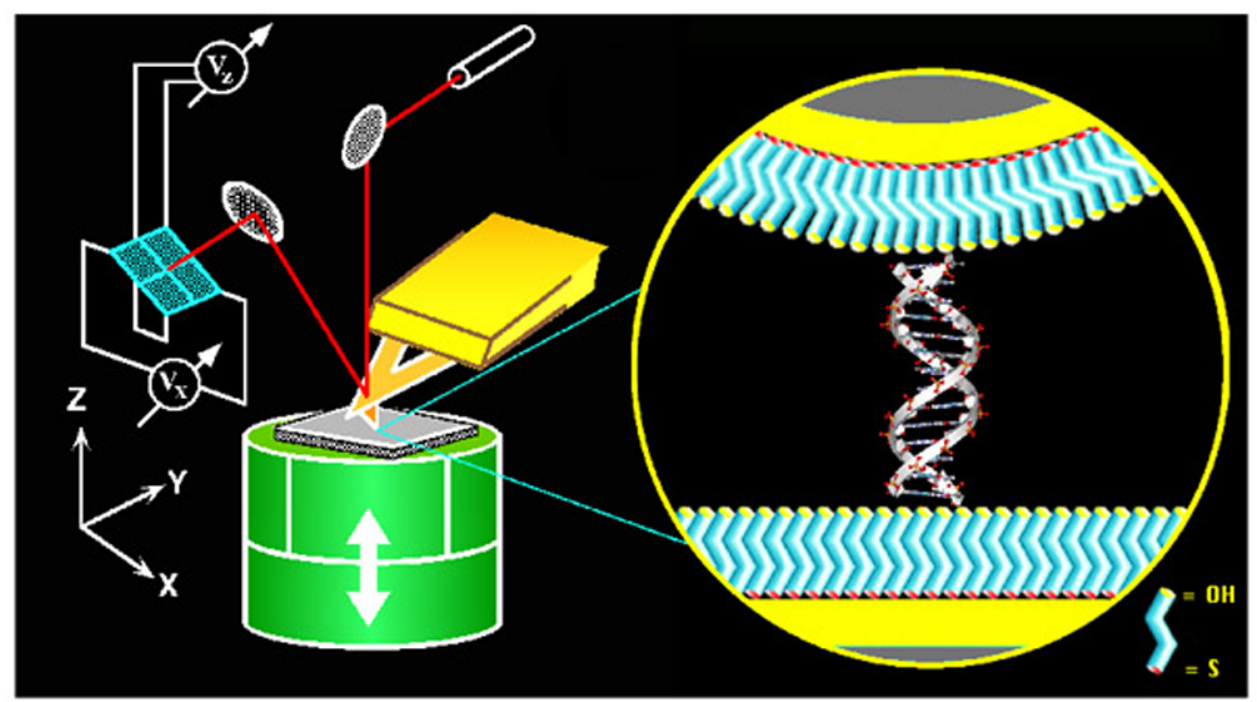

Figure 1: Schematics of a typical molecular force measuring experiment. An AFM tip and sample surface are functionalized with the respective interacting molecules (in this case two complementary DNA strands).

capabilities for measuring and applying molecular level stresses and strains [2]. Both techniques have strengths and weaknesses, for example, optical trapping provides better force resolution, while atomic force microscopy provides better distance control. Overall, atomic force microscopy has an edge for studies of protein-protein and ligand-receptor interactions since these interactions typically generate high forces and steep potential gradients inaccessible with optical trapping techniques. In particular, Chemical Force Microscopy (CFM) allows researchers to study a broad range of specific interactions [11]. In a typical CFM measurement one of the interacting molecules is covalently bound to the tip of the AFM cantilever and another molecule attaches to the sample surface (Figure 1). The technique uses piezoelectric scanners to control the sample position with angstrom-level precision, while AFM cantilever acts as a force sensor, as well as force transducer that loads the bond.

Some of the first attempts to use AFM for measuring specific chemical interactions date back to 1994 when Lieber and co-workers demonstrated that AFM can distinguish between different types of specific chemical interactions between basic functional groups [5]. During the same year Gaub and co-workers demonstrated first measurement of the interaction between biological molecules [4]. The spectrum of molecules probed by the AFM techniques has since expanded to include a large 
number of protein-protein and protein-ligand pairs, DNA, colloidal particles and other chemical systems [3].

\subsection{Noncovalent chemical bond dynamics under external load}

We now need to consider how loading by an external spring changes the bond behavior. We assume that the cantilever behaves as an ideal Hookean spring represented by a parabolic potential. Addition of the cantilever potential to the bond potential, leads to the emergence of a secondary minimum on the potential energy surface, which typically represents the unbound state. The dynamics of the system are then determined by the transition from the primary minimum to this secondary minimum. Let us consider how external loading force changes the thermodynamics and kinetics of the transition between bound and unbound states. In the most general case, the dynamics of this two-well system involves two elementary first order processes unbinding and rebinding with each process characterized by a rate constant. As Bell showed in his pioneering work [], loading the system in the direction of unbound state lowers the barrier to unbinding and simultaneously raises the barrier to rebinding. Consequently, loading leads to the amplification of unbinding rate constant, $\mathrm{k}_{u n b}$, and retardation of the rebinding rate constant, $\mathrm{k}_{r e b}$ :

$$
\begin{aligned}
& k_{u n b}(F)=k_{u n b} \cdot e^{\frac{F \cdot x_{\beta}}{k_{B} T}} \\
& k_{r e b}(F)=k_{r e b} \cdot e^{\frac{-F \cdot x_{\beta}}{k_{B} T}}
\end{aligned}
$$

where $x_{\beta}$ denotes the distance to the transition state from the bottom of the primary well and is the corresponding distance from the bottom of the secondary, cantilever-induced potential well. One of the key points is that the distance is mostly determined by the cantilever potential, which can cause surprising variations in unbinding dynamics. Stiff springs place the secondary well relatively close to the transition barrier, while shallow potentials of weak springs place the secondary minimum far away. Equation 25 then predicts that the rebinding process becomes extremely slow when the bond is stretched by a soft spring. Another key point is that in most CFM experiments the loading force rarely stays constant during the loading process and almost always ramps up linearly, as the piezo scanner retracts from the surface at a constant speed. A simple qualitative analysis shows that the unbinding transition can happen in two different regimes, as determined by the rate of bond loading. Under slow loading of the bond, the unbinding proceeds as an equilibrium process and the force necessary to break the adhesive junction is simply determined by the energy balance between bound and unbound states. Alternatively, if the loading rate is comparable with the rate of at least one of the processes described 
by the Equations 2 and 1, the system never reaches equilibrium and the unbinding proceeds under kinetic control. One direct consequence of this conclusion is that the unbinding force becomes very dependent on how the bond is loaded; in other words, the loading history starts to play a critical role. Before we consider these regimes in more details, we note that equilibrium unbinding imposes extremely restrictive conditions on the system. In particular, to achieve equilibrium we need to load the bond slower than the rebinding rate - the situation, which does not happen often due to the exponential retardation of the rebinding rate by the external load. The Equation 2 indicates that for realistic loading rates the best chance to observe the equilibrium unbinding is with the systems that feature deep bond potentials (i.e. large kreb0) and short distances from the secondary well to the transition state. In practical terms, it means that we can observe equilibrium unbinding only in multibond systems stretched slowly by stiff springs, although even in this case the system should transition into the non-equilibrium unbinding regime with increase of the loading rate. As a general rule, most of the single-molecule bond measurements always happen in non-equilibrium regime.

For the non-equilibrium unbinding we have to distinguish between two different scenarios: unbinding events that occur in near-equilibrium conditions and unbinding events that occur very far from equilibrium. In the first case, the main effect of the departure from the equilibrium is introduction of the energy dissipation. in the second case the bond loading is so fast that it completely suppresses the rebinding and profoundly changes the whole dynamics of the unbinding process. We will now describe the physics of unbinding in these systems in more details, as well as try to focus on the practical system and the force spectroscopy techniques which can extract equilibrium potentials in those situations.

\subsection{Unbinding far from equilibrium: Tethered ligand-receptor sys- tem}

Experimental measurements of the intermolecular interactions often encounter problems associated with non-specific interactions of the tip functional groups with the sample surface. Even if passivating the surfaces with adhesion-resistant functional groups such as PEG [12] or -OH [10] can alleviate this problem, none of these techniques can remove the effects of short-range Van der Waals attraction force between the tip materials and the surface [1]. A popular and effective solution to non-specific interaction interference is to attach the molecules of interest to molecular-size tethers hanging off the tip and surface (Figure ??A). In effect, the tethers "clean up" the system by spatially separating the specific interactions from the non-specific interactions. Another important role of the tethers is to allow maximum conformational freedom to the interacting molecules, thus ensuring a good bond between the 
ligands.

However, effective separation requires tethers which are at least several nanometers long. Since the typical persistence length of the polymer linker is at least an order of magnitude smaller than this length, tether effectively introduces a weak entropic spring in series with the cantilever spring. This second spring changes the energy landscape dramatically (Figure ?? shows a comparison of the energy landscapes between of a tethered and non-tethered system stretched by the same cantilever spring). First of all, the potential that stretches the bond now has two regions- the initial shallow region that corresponds to the stretching of the elastic tether and the steeper region that corresponds to the bending of the cantilever (when the tether is already stretched taught). Second, the unbound state is now separated from the bound state by a very large distance along the stretching coordinate. The main effect of this separation is that the applied load now retards unbinding much stronger (See Eq. 2). It is safe to say that for realistic tether length and stiffness, and for realistic loading forces, the rebinding is completely suppressed. Therefore, unbinding in tethered systems always presents a kinetic problem and we need to analyze it as such.

Yet, before we describe this analysis we should consider the qualitative physics of the unbinding under the tethered ligand potential. If we are stretching the tether slowly relative to the intrinsic bond dissociation time, then the work to unbind the bond is done mostly by the tether spring. This is the situation often encountered in the optical tweezers measurements where researchers use weak entropic springs, such as DNA, to apply forces to molecular motors and proteins. Typical AFM experiment deals with stronger (i.e. longer-living) bonds and stretches them much faster than necessary for this scenario; therefore, in the AFM measurements the tether is stretched out before the bond starts breaking. Consequently, the system transitions to the steeper Hookean part of the potential and that is where the actual unbinding occurs. The paradoxical conclusion of this analysis is that for fast forceassisted unbinding a tethered systems is equivalent to a non-tethered system, i.e. we can analyze it as if the tether does not even exist! In fact, the only role of the tether is to suppress rebinding. Despite the complications commonly associated with the tether elasticity, tethered ligand pairs, in fact, are an ideal experimental system to study the kinetics of the molecular bond rupture under the external loading force.

Evan Evans analyzed such kinetics in 1997 [?] by applying classic Kramers' version of the transition state theory to the case of loading an overdamped system with a weak external spring in absence of rebinding processes. As we mentioned before, thermal fluctuations will eventually drive the system out of potential well even in absence of any loading force. However, application of the external force exponentially amplifies this process, and therefore drastically shortens the bond lifetime. If we mimic the experimental conditions by assuming that force increases 
constantly during the experiment (as we keep moving the AFM piezo away from the surface and keep bending the cantilever), we can correlate the unbinding event to a particular loading force that will define the measured bond strength. For linear loading Evans model predict a remarkable result that the measured loading force is proportional to the logarithm of the loading rate:

$$
f=\frac{k_{B} T}{x_{\beta}} \ln \frac{k_{B} T}{R_{f} \tau_{0}}
$$

Evans and other have observed the scaling predicted by this equation in several experimental systems [?]. As we discussed, this analysis should be fully applicable to the tethered ligand case, with the only caveat that we would need to use correct loading rate. We will discuss our use of Monte-Carlo simulations to test this assumption in the Results section.

\subsection{Near-equilibrium unbinding: Jarzynski equality and reconstruc- tion procedure}

If we use force to break a bond in near-equilibrium condition(i.e. where the rebinding is slow but not yet negligible), the energy dissipation during unbinding will lead to higher forces along the unbinding trajectory and overestimate the overall work. In the past dissipation frustrated most attempts to determine true thermodynamic potential energy. However, recently C. Jarzynski discovered a remarkable equality [7] that connected the results of non-equilibrium measurements to the equilibrium potential (Equation 4).

$$
\left\langle e^{-\frac{W_{i}}{k_{B} T}}\right\rangle=e^{\frac{-\Delta G}{k_{B} T}}
$$

Hummer and Szabo recently adapted Jarzinski formalism to potential energy reconstructions. They showed a theoretical proof that Jarzynski equality can recover the equilibrium interaction potential through the Boltzman-weighting of the integrated work values [6]:

$$
\Delta G=-k_{B} T \ln \left\langle e^{-\frac{\int_{t} F(z) d z-\frac{F(0)^{2}}{2 k}}{k_{B} T}}\right\rangle
$$

The integration in the Equation 5 is performed along the stretching trajectory, $t$, and the second term in the exponent represents the weighting of the trajectory by the initial cantilever deflection. 
Jarzynski equality (Equation 4) has a deceptively simple physical explanation. The exponential weighting gives a disproportionately large weight to those trajectories where thermal fluctuations help the system to move along the reaction path and where the total work is smaller than the equilibrium energy difference; the complete average can thus recover the equilibrium work value. Bustamante and co-workers, who pioneered applications of Jarzynki equality to biophysics [9], pointed out that this reliance on the fluctuations immediately restricts any practical applications of the Jarzynski equality to the microscopic processes, where the magnitude of the thermal fluctuations is significant. Moreover, even if Jarzynski equality applies to all non-equilibrium process, practical considerations restrict its application to processes that are relatively close to the equilibrium. If the unbinding happens too far from equilibrium, collecting enough data to make the averaging work simply becomes impractical. Unfortunately, this consideration places the practicality of the Jarzynski equality application to most tethered systems in serious doubt. We investigate this question in the Results section, as well as demonstrate a system where Jarzynski equality does work.

\section{Results}

\subsection{Monte-Carlo simulation of a tethered ligand-receptor system}

To obtain an accurate picture of the interaction dynamics in a tethered system it is extremely important to have access to a variety of different configurations featuring different linker lengths, elasticities and different protein-protein binding strengths. Synthetic challenges made it unrealistic to expect to probe a large number of different experimental systems over a short duration of this project. Therefore, we decided to use a simple physical model [?] to conduct Monte-Carlo style simulations of the unbinding dynamics under external loading which simulated condition of the AFM experiment.

Our model features a one-well potential barrier, which represents the proteinprotein bond, connected to an entropic spring, representing the linker (Figure 2). The model uses a very small set of parameters to describe the system: intrinsic unbinding rate for the protein-protein bond $\left(U_{0}\right)$, potential well width $\left(x_{\beta}\right)$, and linker contour length $(C)$ and persistence length $(L)$. Initially, we will use the Inextensible Worm-Like-Chain (WLC) model to describe the linker elasticity; later we can expand the range of elasticity models to include Extensible Worm-Like Chain model and a Freely-Jointed Chain model. Those models may be better suited for describing the entropic elasticity of a PEG linker. We will then simulate the force unbinding of the system by moving the AFM tip end away from the sample at a constant rate. 

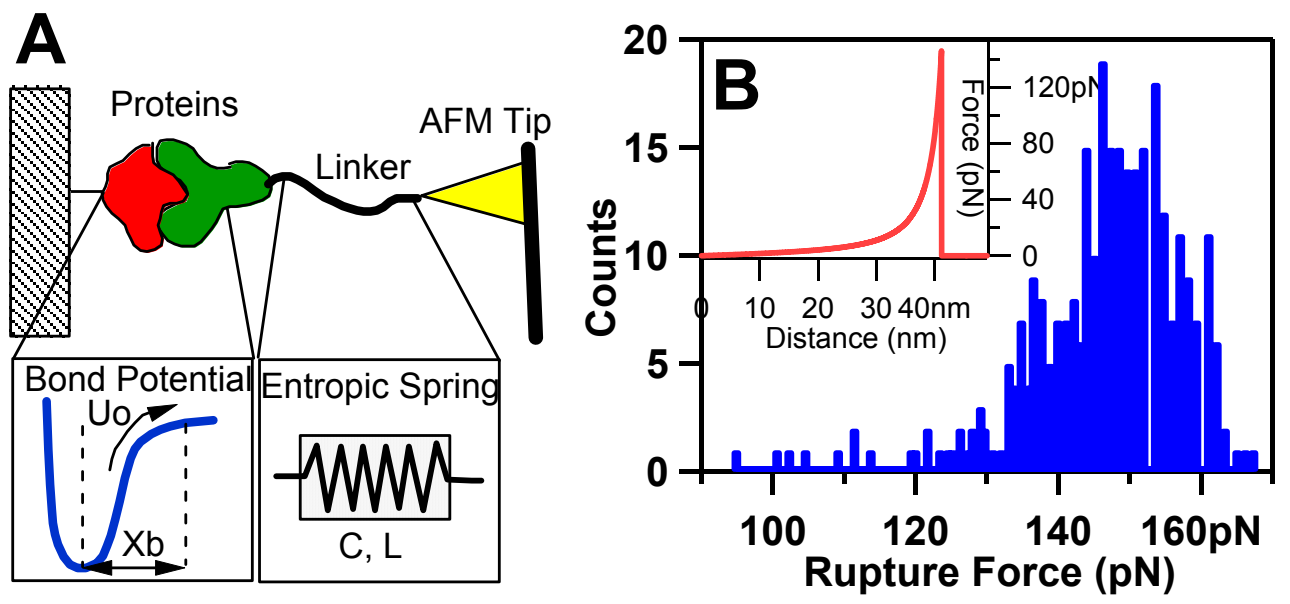

Figure 2: A. Schematics of the model for Monte-Carlo simulations showing model parameters. B. A distribution of the binding forces that we obtained for a test run that features 300 individual simulated pulls. The inset shows a force vs. distance trace obtained in one of the pulls

We assume that the dissociation rate of a receptor-ligand pair is described by the Arrhenius model for bond lifetime:

$$
\alpha_{o}=\omega e^{-\Delta G^{*} / k_{B} T}
$$

Using Bell's approximation, the addition of a force has the effect of lowering the activation barrier $\Delta G^{*}$ by $F x_{\beta}$. Here $x_{\beta}$ is the distance from the minimum of the well, to the maximum of the energy barrier. We then get an unbinding rate as a function of applied force:

$$
\alpha(F)=\omega e^{-\left(\Delta G^{*}-F x_{\beta}\right) / k_{B} T}=\alpha_{o} e^{F x_{\beta} / k_{B} T}
$$

The force we are applying with the cantilever is transferred to the bond through a polymer linker. If this polymer is described by the WLC model, we can use the following relationship between force and extension:

$$
F(x)=\frac{k_{B} T}{A}\left(\frac{1}{4(1-x / L)^{2}}-\frac{1}{4}+\frac{x}{L}\right)
$$

We perform the simulation according to the following procedure: At each interval time step $\Delta t$ we calculate an extension from $x=x_{o}+v \Delta t$. Where $v$ is the velocity 
we move the cantilever away from the surface. This extension is entered into the Equation $=8$ to calculate a force. Using Equation 7 we then find the probability of bond rupture from:

$$
d P=\alpha(F) \Delta t
$$

That is, in the interval $\Delta t$ the probability of a rupture event is given by Equation 9. We then choose a random number 0 and 1 and compare it to $d P$. If the random number is less than $d P$ then the bond has broken and we set the applied force to zero for the remainder of the extension process. If it is greater than $d P$ then we continue to apply force by stretching the linker further.

As we ran this simulation multiple times, we built up a histogram of the rupture forces, which simulated experimental results. Figure 3 shows the dependence of the expected dynamic force spectra on the length of the tether. Noticeably, the tether shift the dynamic force spectra; yet the slope of the spectra remains the same. Therefore, we concluded that the main effect of the tether presence is to change the loading rate. To counteract this effect we have used a different strategy for data processing where we used an effective loading rate determined from the pulling trace region right before the bond rupture event (see section on Muc1-Antibody measurements). We are currently running more sophisticated simulation on potential energy surfaces corresponding to the rupture of two tethered ligands (see an example of such surface on the cover of this report).

\subsection{Equilibrium energy profile reconstruction for a short DNA du- plex}

AFM experiments typically use stiffer probes that help to avoid cantilever instabilities that arise when the gradient of the interaction potential exceeds cantilever stiffness. This requirement lead to relatively high loading rates which greatly increased the energy dissipation during the experiment and place the measurements in non-equilibrium regime. In fact, AFM measurements almost always involve energy dissipation, which until recently precluded their use for extraction of equilibrium binding energy information. We have recently tested this approach by applying it to the results of the AFM measurements of DNA unbinding (Figure 4A). We demonstrated that Boltzmann-weighted averaging algorithm indeed recovers equilibrium melting enthalpy (to within less than 5\%!), and true force vs. extension profile (Figure 4B). For comparison, simple averaging of the work over the force curve cycle overestimates the binding enthalpy by $40 \%$. This example also highlights another important feature of this technique: it allows us to estimate the energy dissipation in the unbinding cycle by comparing the actual force profile with the equilibrium force profile. 


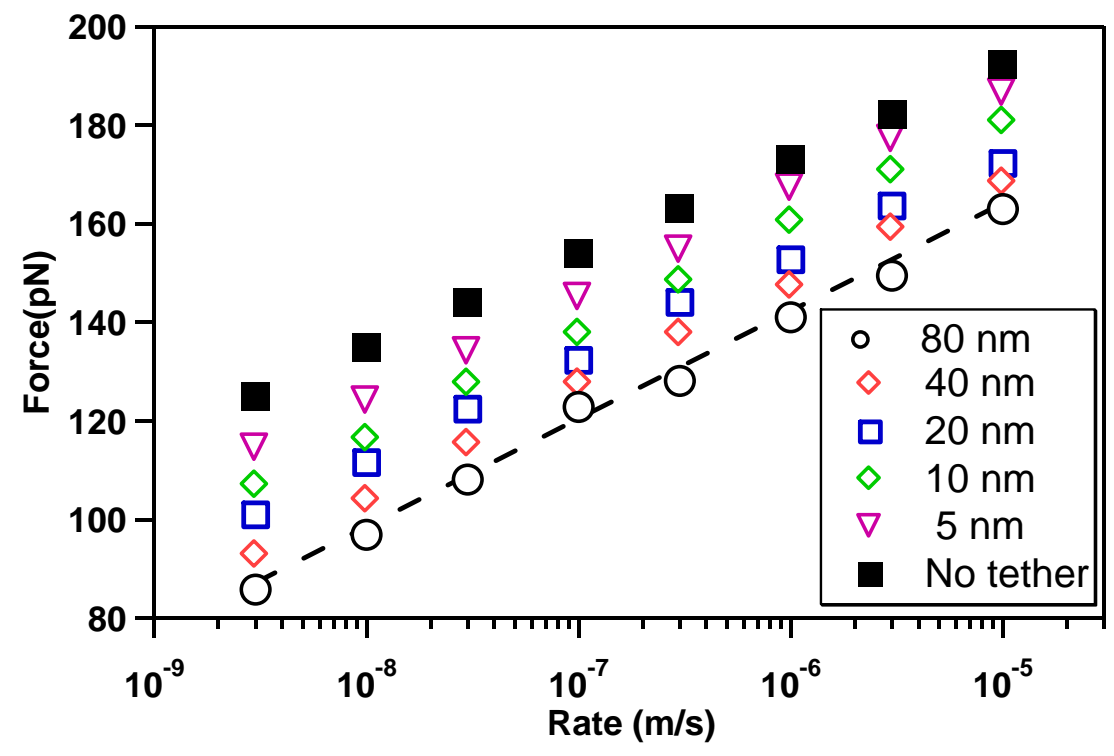

Figure 3: Dynamic force spectra showing most probable rupture forces as a function of loading rates, calculated for different tether lengths.

\subsection{Dynamic Force Spectroscopy of Mucin 1 interactions with sin- gle and multiple antibodies}

Mucin 1 (Muc1) is an important protein which is often overexpressed on the surface of cancer cells. This property makes it an effective target for several types of prototype cancer drugs. Radioimmunotherapy approach uses a complex of an abMuc1 antibody and a potent $\alpha$-particle emitter. The antibody binds with high affinity to the Muc1 proteins on the cancer cells and delivers the radioactive payload which destroys the cell.

To establish the feasibility of detecting multivalent interactions with dynamic force spectroscopy we used this technique to measure the kinetic off-rates for mono, bi-, and tri-valent binding of antibodies to Muc1 surface target in an in-vitro model system. These studies used monoclonal antibody fragments specific to Muc1 available from the RIT drug design effort at the DeNardo group at the UC Davis Cancer Center. Our group has also utilized experimental techniques and approaches that we have developed previously for measurement of intermolecular interactions using force spectroscopy. 

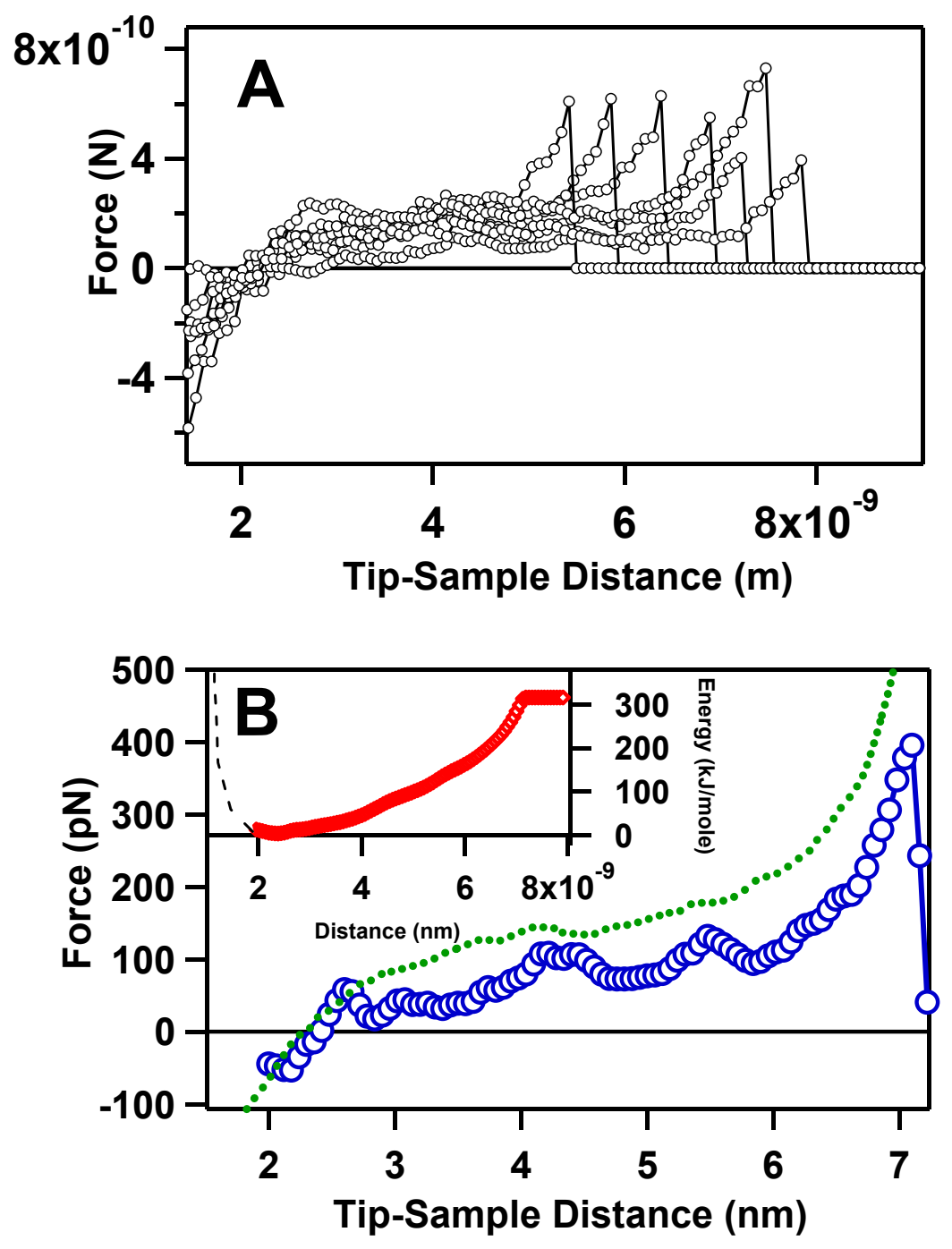

Figure 4: A. Seven individual force vs. distance traces for unbinding of 14-mer DNA duplex (data taken from Ref.[10]). B. Force vs. distance traces obtained by averaging DNA stretching traces in $\mathbf{A}$ using simple averaging (dotted line) and weighted work integral averaging as described by Equation 5(circles). The inset graph shows the interaction potential reconstructed by using Equation 5. The part of the potential corresponding to the repulsive wall was not reconstructed and is shown by the dashed line as a guide to the eye). 


\subsubsection{Design and preparation of an in-vitro model system for force spec- troscopy of multivalent binding of tethered antibodies targeting Muc1}

We have mimicked the targeting part of the RIT drug by coupling purified scFv antibody fragments to flexible PEG tethers that linked the antibodies to the AFM tip. We attached the Muc1 peptide to the surface of a self-assembled monolayer to mimic the surface of a cancer cells. To provide the Muc1 and a target antibody with the maximum binding flexibility we chose to attach the peptide to the monolayer surface via a flexible PEG linker (Figure 5, inset). The DeNardo group has generated scFvs by pCANTAB $5 \mathrm{E}$ phage display/expression and genetically engineered them to express an additional C-terminal cysteine group (scFv-Cys), which does not interfere with the binding domain (Albrecht et al. 2004). ScFv expression from pCANTAB $5 \mathrm{E}$ Cys allowed production of soluble scFv-Cys protein from E. coli. Our group then coupled scFv-Cys protein to a PEG-NHS-maleimide scaffold to form PEG(scFv) conjugates. These conjugates, as well as the 100-amino-acid long Muc1 peptide fragment (Peptide Synthesis Facility, U. Pittsburgh) coupled to the NHS-PEG linker, were then attached to the surfaces of self-assembled monolayers on the surfaces of our probe and sample using protocols established previously by our colleagues at LLNL (Ratto et al. 2004). The average length of each of our PEG linkers was $252.8 \mathrm{~nm}$, as established by mass-spectrometric measurement of molecular weight. To control the protein attachment density to the AFM tip and sample surface we diluted the reactive PEG with an inert "spacer" PEG. We tuned the dilutions in the tip and sample preparation protocols differently to limit the number of the tethered antibodies on the tip to only a few, while providing a relative abundance of Muc1 target on the surface.

\subsubsection{Detection of specific antibody-Muc1 binding}

We have measured specific interactions by collecting a series of the force vs. distance traces as we approached and withdrew the AFM cantilever from the surface in a 20 $\mathrm{mM}, \mathrm{pH} 7.0$ phosphate buffered saline solution. Figure ?? shows a representative force vs distance trace from such experiment. The trace shows a series of abrupt jumps in the cantilever deflection corresponding to breakages of specific and nonspecific tip-sample linkages as the tip withdrew from the surface. Each rupture was preceded by a gradual cantilever tension increase corresponding to the stretching of the tether linkage. We have confirmed this assignment by showing that these regions

fit very well the theoretical model describing PEG elasticity (Figure 6). Use of the defined tether lengths in our system allowed us to assign the breakage events that typically happen at tip-sample separation corresponding to the maximum combined 


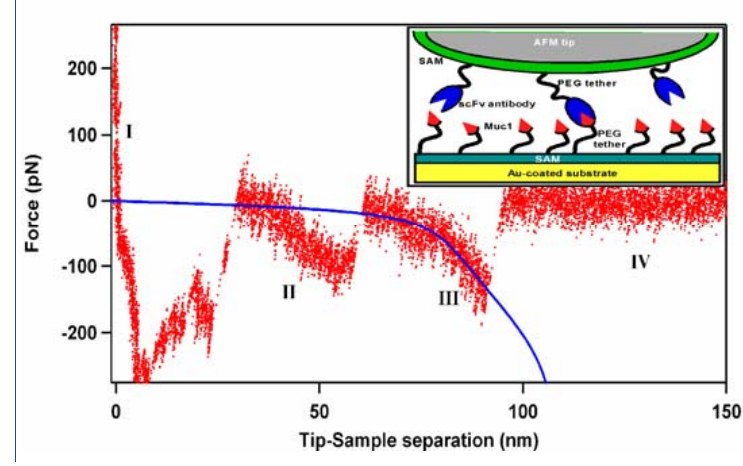

Figure 5: Representative force vs. separation trace obtained for interactions between scFv fragment and Muc1 peptide. Different regions on the force curve correspond to: (I) hard tip-surface contact, (II) non-specific interaction, (III) stretching of a PEG tether, and (IV) free cantilever deflection away from the surface. Inset shows the detailed experimental geometry.

tether extension for the Muc1 and the antibody to the rupture of the specific Muc1antibody bond. The rupture events observed at tip-sample separations shorter than two tether lengths correspond to non-specific interactions in the system. The specific binding events typically occurred at longer distances and exhibited rupture forces tightly clustered around $150 \mathrm{pN}$, while non-specific interactions produced lower rupture forces. Occasionally these specific binding events also exhibited higher binding forces, which correspond to the rupture of multiple antibody-Muc1 bonds.

We have verified that we detected specific Muc1-antibody interactions by comparing binding forces recorded in absence and in presence of the excess of free solution-phase Muc1 peptide, which could block the antibody binding sites. A comparison of these two experiments (Figure 6) clearly shows that the only the specific interactions peak vanished when the antibody was blocked by the excess of free Muc1; in contrast, non-specific interaction peaks were not affected.

\subsubsection{Force spectroscopy determination of kinetic off-rates for mono-, bi-, and tri-valent tethered antibodies}

Measurement of the rupture forces in molecular assemblies as a function of the loading rate can provide the information about the affinity of the interaction (Evans 1999; Zepeda et al. 2003; Patel et al. 2004). Unlike our previous dynamic force spectroscopy measurements (Zepeda et al. 2003), our tethered antibody system incorporates non-linear entropic springs (the tethers), and the loading rate is no 


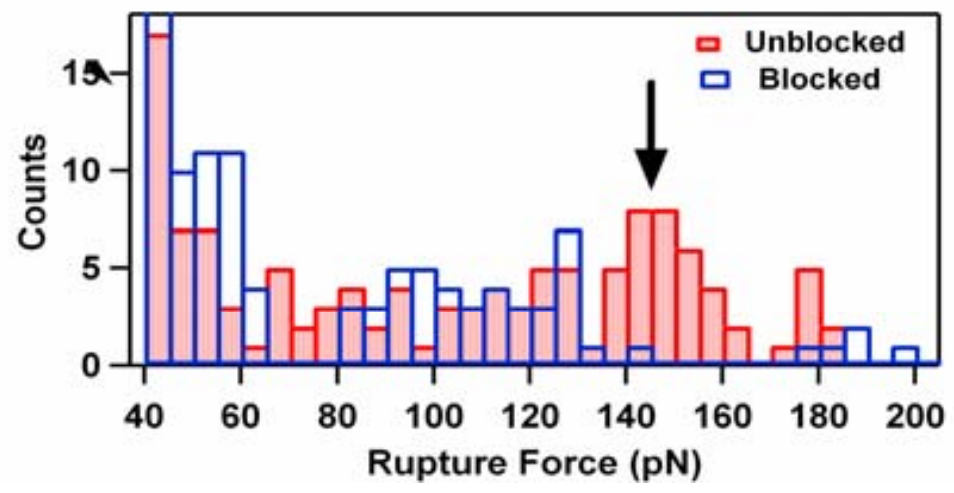

Figure 6: Histograms of the rupture forces in absence (unblocked) and in presence (blocked) of the excess of solution-phase Muc1.

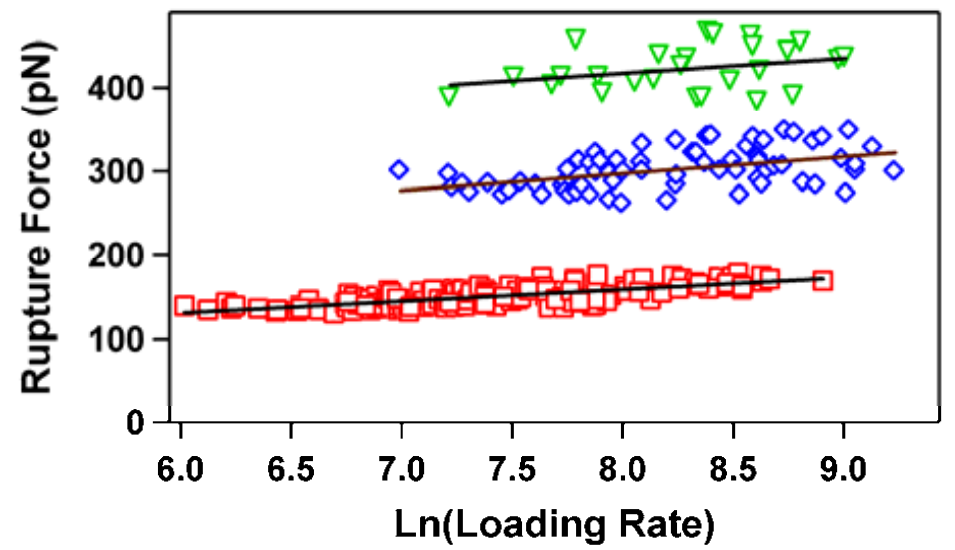

Figure 7: Dynamic force spectra corresponding to the rupture of one (red), two (blue), and three (green) antibody- Muc1 pairs. 


\begin{tabular}{|c|c|c|}
\hline Valency & $\mathrm{k}_{\text {off }}$ & $\mathrm{x}_{\beta}, \AA$ \\
\hline 1 & $3.6 \cdot 10^{-3}$ & 2.8 \\
2 & $7.1 \cdot 10^{-5}$ & 2.0 \\
3 & $4.4 \cdot 10^{-9}$ & 2.4 \\
\hline
\end{tabular}

Table 1: Mon- and multi-valent Muc1-antibody interaction parameters determined from the dynamic force spectra

longer solely determined by the speed of the AFM cantilever loading. Fortunately, Gaub and coworkers showed that similar analysis can be extended to tethered systems by determining the effective loading rate from fitting the extension profiles to an appropriate polymer elasticity model (Friedsam et al. 2003). Indeed, when we plotted our data as a function of this effective loading rate (Figure 7 , lower trace) we obtained a characteristic logarithmic dependence of the binding force on the loading rate predicted by the Equation B.1. This dependence allowed us to determine the kinetic off-rate for a Mucl-antibody bond to be $3.6 \cdot 10^{-3} \mathrm{~s}^{-1}$, which is in good agreement with the available SPR data.

In addition to the single protein-protein binding events, force spectroscopy allowed us to determine interactions parameters for the rupture of multiple Muc1antibody pairs. A number of extension traces did not fit to the stretching profile of a single PEG tether, yet they fit remarkably well the stretching profiles for two or three PEG tethers connected in parallel. Notably, the theory predicts that such parallel bond configurations should produce dynamic force spectra with identical slopes corresponding to the common distance to the transition state; yet the spectra should be shifted along the vertical axis to reflect lower kinetic off-rates for higher order binding. Dynamic force spectra (Figure 7) that we obtained for single and multiple Muc1-antibody interactions are in remarkable agreement with this prediction. To our knowledge this is the first demonstration of the force spectra of multiple identical bonds in parallel configuration. These data allowed us to start quantifying the advantage provided by multivalent binding. Table 1 lists the kinetic off-rates and the distances to the transition state determined from our data. It is clear that addition of Muc1-binding antibody units results in progressive reduction of the kinetic off-rate, which is one of the critical parameters controlling drug retention time in tumors. Significantly, force spectroscopy allowed clean separate determination of kinetic stability for single and multiple Muc1-antibody bonds. 


\section{References}

[1] P. D. Ashby, L. W. Chen, and C. M. Lieber. Probing intermolecular forces and potentials with magnetic feedback chemical force microscopy. J. Am. Chem. Soc., 122(39):9467-9472, 2000.

[2] C. Bustamante, J. C. Macosko, and G. J. L. Wuite. Grabbing the cat by the tail: Manipulating molecules one by one [review]. Nature Reviews Molecular Cell Biology, 1(2):130-136, 2000.

[3] H. Clausen-Schaumann, M. Seitz, R. Krautbauer, and H. E. Gaub. Force spectroscopy with single bio-molecules. Curr Opin Chem Biol, 4(5):524-530, 2000 .

[4] E. L. Florin, V. T. Moy, and H. E. Gaub. Adhesion forces between individual ligand-receptor pairs. Science, 264(5157):415-17, 1994.

[5] C. D. Frisbie, L.F. Rozsnyai, A. Noy, M.S. Wrighton, and C.M. Lieber. Functional group imaging by chemical force microscopy. Science, 265:2071, 1994.

[6] G. Hummer and A. Szabo. Free energy reconstruction from nonequilibrium single-molecule pulling experiments. Proc Nat Acad Sci USA, 98(7):3658-3661, 2001.

[7] C. Jarzynski. Nonequilibrium equality for free energy differences. Phys. Rev. Lett., 78(14):2690-2693, 1997.

[8] C. Jeppesen, J. Y. Wong, T. L. Kuhl, J. N. Israelachvili, N. Mullah, S. Zalipsky, and C. M. Marques. Impact of polymer tether length on multiple ligandreceptor bond formation. Science, 293(5529):465-468, 2001.

[9] J Liphardt, S Dumont, SB Smith, I Tinoco Jr, and C Bustamante. Equilibrium information from nonequilibrium measurements in an experimental test of jarzynski's equality. Science, 296:1832-1835, 2002.

[10] A Noy, DV Vezenov, JF Kayyem, TJ Meade, and CM Lieber. Stretching and breaking duplex dna by chemical force microscopy. Chem Biol, 4(7):519-527, 1997.

[11] A Noy, DV Vezenov, and CM Lieber. Chemical force micrtoscopy. Ann. Rev. Mat. Sci., 27:381-421, 1997.

[12] K. L. Prime and G. M. Whitesides. Adsorption of proteins onto surfaces containing end-attached oligo(ethylene oxide) - a model system using selfassembled monolayers. J. Am. Chem. Soc., 115(23):10714-10721, 1993. 
[13] J Y Wong, T L Kuhl, J N Israelachvili, N Mullah, and S Zalipski. Direct measurement of a tethered ligand-receptor interaction potential. Science, 275:820$822,1997$.

[14] Hiromi Yamakawa. Modern theory of polymer solutions. Harper Row, New York,, 1971. illus. $25 \mathrm{~cm}$. 